\title{
Parafusos pediculares no tratamento da cifose de Scheuermann: resultados e complicações*
}

\author{
Pedicle screws in the treatment of Scheuermann's \\ kyphosis: results and complications
}

\author{
Rodrigo D'Alessandro de Macedo ${ }^{1}$, MÁrcio Fim², Bruno Pinto Coelho Fontes ${ }^{3}$
}

\section{RESUMO}

O tratamento cirúrgico da cifose de Scheuermann permanece um tópico em debate. Tratamento tradicional tem incluído liberação anterior associada à fusão dos segmentos discais apicais, seguida por instrumentação posterior. Objetivo: Avaliar se o uso do sistema de parafusos pedicular vertebral promove melhor estabilização e correção da deformidade, sem procedimento compressivo, diminuindo os risco de complicações. Métodos: Foram avaliados 19 pacientes com cifose de Scheuermann, que foram submetidos à liberação e fusão anterior por

Trabalho realizado no Hospital Governador Israel Pinheiro HGIP/ IPSEMG, Belo Horizonte (MG), e no Hospital da Baleia, Belo Horizonte (MG).

1. Preceptor do Grupo de Coluna do Hospital da Baleia; Coordenador do Grupo de Coluna do Instituto de Previdência dos Servidores de Minas Gerais - IPSEMG; Mestre em Ciências e Técnicas Nucleares pelo Departamento de Engenharia Nuclear da Universidade Federal de Minas Gerais - UFMG - Belo Horizonte (MG), Brasil.

2. Médico Residente do 3o ano de Ortopedia e Traumatologia do Hospital Governador Israel Pinheiro - HGIP/IPSEMG - Belo Horizonte (MG), Brasil.

3. Preceptor do Grupo de Coluna do Hospital da Baleia - Belo Horizonte (MG), Brasil.

Endereço para correspondência: Rua dos Sabiás, 1.994, Estância Serrana - 34000-000 - Nova Lima, MG. Tel.: (31) 3344-2777.

E-mail: rodrigodalessandro@terra.com.br

Recebido em 22/10/07. Aprovado para publicação em 24/1/08.

Copyright RBO2008 toracotomia aberta, seguida por instrumentação posterior com sistema de parafuso pedicular vertebral posicionados pela técnica de mão livre. Préoperatoriamente, os pacientes foram analisados pela escala analógica visual (EVA) de dor e radiografias. No pós-operatório, foi analisado o grau de correção da curva, complicações, EVA para dor e nível de satisfação com a cirurgia. A média de seguimento foi de 37,5 meses (12,6-61,7 meses). Resultados: Cifose pré-operatória variou de $66^{\circ}$ a $94^{\circ}$, com média de $77,6^{\circ}$, e média da EVA de 6,6 pontos. No pósoperatório a cifose média foi de $35,8^{\circ}$ (variação de $2^{\circ}$ a $5^{\circ}$ ), sendo obtida média de correção de $53,8 \%$. A EVA foi de 0,6 ponto, com todos os pacientes, exceto um, satisfeitos com o resultado da cirurgia. Dentre as complicações: soltura de dois parafusos em um paciente, com necessidade de reintrodução e extensão da instrumentação, um paciente com infecção superficial resolvida clinicamente, uma quebra assintomática de parafuso e um paciente com infecção tardia resolvida com a retirada do implante. Conclusão: O tratamento cirúrgico da cifose de Scheuermann, usando liberação e fusão anterior por toracotomia aberta seguida por instrumentação posterior com sistema de parafuso pedicular vertebral por meio da técnica de mão livre, mostrou-se, nessa série de pacientes, segura e eficiente.

Descritores - Doença de Scheuermann; Cifose; Deformidade/coluna vertebral; Fusão espinhal; Toracotomia; Escala visual analógica de dor 


\section{ABSTRACT}

Surgical treatment of Scheuermann's kyphosis is still subject to debate. Traditional treatment has included an anterior release associated to fusion of apical disk segments, followed by posterior instrumentation. Objective: To evaluate if the vertebral pedicle screw system yields a better stabilization and correction of the deformity, without a compressive procedure, thus decreasing the risk of complications. Methods: 19 patients with Scheuermann's kyphosis were evaluated after being submitted to anterior release and fusion by open thoracotomy followed by posterior instrumentation with a vertebral pedicle screw system with the screws positioned with the free-hand technique. Before surgery, the patients were analyzed according to the visual analogical pain scale (EVA) and using X-rays. After surgery, analysis was made of the extension of the curve correction, complications, EVA for pain, and level of satisfaction with the surgery. Mean follow-up was 37.5 months (12.6 - 61.7 months). Results: Preop kyphosis varied from $66^{\circ}$ to $94^{\circ}$, with a mean of $77.6^{\circ}$ and the mean EVA was 6.6. After surgery, the mean kyphosis was $35.8^{\circ}$ (ranging from $23^{\circ}$ to $50^{\circ}$ ), a mean correction of $53.8 \%$ being obtained. EVA was 0.6 , and all patients but one, were pleased with the result of the surgery. Complications: two screws were detached in one patient and had to be reintroduced and instrumentation had to be expanded, one patient had superficial infection that was clinically solved, a screw broke asymptomatically, and one patient had late infection that was cured with the removal of the implant. Conclusion: Surgical treatment of Scheuermann's kyphosis using anterior release and fusion by open thoracotomy followed by posterior instrumentation with a vertebral pedicle screw system using the free hand technique showed, in this series, to be safe and efficient.

Keywords - Scheuermann's disease; Kyphosis; Deformity/ spinal column; Spinal fusion; Thoracotomy; Visual analogical pain scale.

\section{INTRODUÇÃO}

Scheuermann descreveu uma alteração vertebral juvenil cifótica que poderia ser distinguida da cifose astênica (postural) com base na rigidez peculiar. Ele observou que a deformidade era causada por um acunhamento de corpos vertebrais e também identificou alterações características do corpo vertebral associadas ao achatamento $^{(1)}$.

Sorensen sugeriu que a definição de cifose de Scheuermann (CS) deveria ser uma cifose incluindo três vértebras adjacentes centrais com acunhamento de cinco ou mais graus ${ }^{(2)}$. Entretanto, essa definição foi questionada pela observação de formas atípicas da doença de Scheuermann (hipercifose torácica, rígida sem acunhamento vertebral e alterações vertebrais sem acunhamento) $)^{(3)}$.

A etiologia da deformidade tem sido atribuída a defeitos das placas terminais de crescimento, osteoporose juvenil, ou causas mecânicas. Alterações têm sido identificadas histologicamente, porém não se sabe se são primárias ou secundárias às alterações da biomecânica ou trauma repetitivo. Portanto, a etiologia permanece desconhecida ${ }^{(3-4)}$.

A prevalência CS varia de 0,4 a $8,3 \%$ da população geral, dependendo se o diagnóstico é baseado em critérios radiográficos ou clínicos ${ }^{(2)}$.

A razão na incidência do gênero masculino/feminino varia na literatura devido ao critério de inclusão variável da população estudada ${ }^{(5)}$.

A decisão de submeter o paciente à cirurgia deve ser avaliada individualmente e após uma discussão entre o cirurgião, o paciente e seus responsáveis. Benefícios do tratamento quanto ao alívio da dor e à melhora da aparência física e auto-estima, bem como suas implicações sociais, devem ser ponderados em relação às complicações eventuais.

Considerando a controvérsia sobre a indicação cirúrgica, foi proposto um estudo prospectivo de uma série consecutiva de pacientes com diagnóstico de CS submetidos à liberação e artrodese anterior por toracotomia convencional seguida por instrumentação com sistema de parafusos pediculares.

O objetivo deste estudo é avaliar a característica da amostra em questão, os resultados do tratamento quan- 
to à melhora do quadro álgico, o grau de correção da deformidade e a satisfação do paciente com o procedimento.

\section{MÉTODOS}

Um total de 19 pacientes submetidos a tratamento cirúrgico de CS no período de fevereiro de 2001 a maio de 2007, no Hospital da Baleia e no Hospital do Instituto de Previdência dos Servidores do Estado de Minas Gerais-IPSEMG, foi incluído no estudo. A distribuição quanto a sexo e faixa etária é apresentada na tabela 1. A média de idade dos pacientes foi de 19,0 anos, sendo 19,6 anos para os do sexo masculino e 17,4 anos para os do feminino.

TABELA 1

Distribuição quanto ao sexo

\begin{tabular}{lcc}
\hline \multicolumn{1}{c}{ Sexo } & Média de idade (anos) & № de pacientes \\
\hline Masculino & 19,6 & 14 \\
Feminino & 17,4 & 5 \\
\hline
\end{tabular}

Foi considerado como critério diagnóstico de doença de Schuermann o paciente com cifose torácica rígida clinicamente acima de $45^{\circ}$ e pelo menos uma vértebra acunhada ${ }^{(6)}$.

Foram excluídos os pacientes com cifoses torácicas flexíveis avaliadas através de exame físico e radiografia em hiperextensão, além de pacientes com outras causas de cifose.

Foram indicados para cirurgia pacientes com curva acima de $50^{\circ}$ associada a dor refrataria ao tratamento clínico e/ou deformidade estética importante.

Todas as cirurgias foram realizadas pela mesma equipe em dois hospitais de Belo Horizonte: Hospital do Instituto de Previdência dos Servidores do Estado de Minas Gerais e Hospital da Baleia.

Os pacientes foram submetidos à liberação anterior por toracotomia convencional e fusão seguida por artrodese posterior com instrumentação posterior com sistema de parafusos pediculares em dois estágios, com média de sete dias entre os mesmos. Em decúbito lateral direito foi realizada toracotomia padrão à esquerda com costectomia, liberação anterior e artrodese sem instrumentação de uma média de sete discos. Discectomia completa foi realizada no máximo de níveis possíveis. Nos espaços discais foi colocado apenas enxerto da costela ressecada.

Após uma média de sete dias, os pacientes foram submetidos ao segundo tempo. Em decúbito ventral, após uma via de acesso posterior e dissecção subperiostal, realizava-se facectomia inferior em todos os níveis. Utilizou-se como ponto de inserção do parafuso pedicular a intersecção da borda superior do processo transverso e a linha vertical lateral da faceta articular superior $^{(7)}$. Por meio de broca de $3,2 \mathrm{~mm}$, com trepano de baixa rotação, e por percepção manual da resistência óssea avançava-se vagarosamente, sempre certificando-se por pequenas incursões a presença de osso no fundo do orifício. Com um palpador maleável confirmava-se a presença de osso nas paredes superior, inferior, medial, lateral e no fundo do trajeto ${ }^{(8)}$. Após essa confirmação, os parafusos eram introduzidos a mão livre (free hand), técnica diferente da relatada por Kim et al, devido à utilização de broca para realização do trajeto ${ }^{(9)}$. Parafusos foram colocados bilateralmente ou unilateralmente em cada nível. A posição dos parafusos era checada por radiografias em perfil e póstero-anterior. As hastes eram previamente moldadas e, através de manobra de "cantilever" fixando as mesmas de distal para proximal nos parafusos, realizou-se a correção da deformidade. Manobras de compressão foram utilizadas no ápice da curva. Usaramse dois dispositivos de tração transversal por paciente. Foram empregados apenas os enxertos locais após a decorticação do arco posterior.

Para seleção dos níveis de instrumentação foi considerado o nível proximal como a vértebra que representava a vértebra de transição da cifose torácica com a lordose cervical, usualmente T2 ou T3, e o nível distal de fusão como a vértebra mais proximal que tocasse a linha vertebral sacral posterior, geralmente L2 ou L3. Foram abordados níveis de T2 a L3 (gráfico 1).

Não foi realizado estudo de monitorização da medula por não dispormos de condições que o permitissem. Todos os pacientes foram submetidos a wake-up test após a redução da deformidade. 


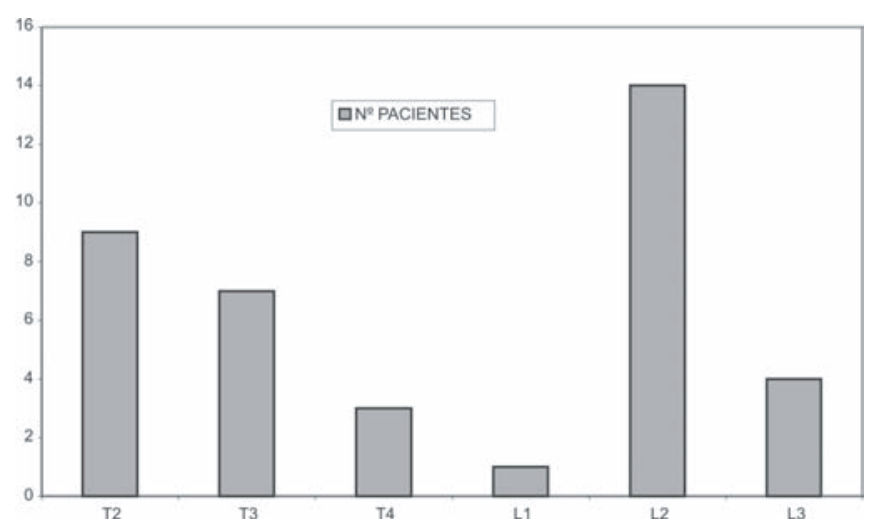

Gráfico 1 - Extensão da artrodese proximal e distal na abordagem posterior

Não foi utilizada órtese no pós-operatório dos pacientes acompanhados neste estudo.

Os pacientes foram acompanhados semanalmente no primeiro mês de pós-operatório e a cada dois meses no primeiro ano. Após, os controles foram feitos semestralmente. As avaliações foram realizadas por membros da equipe e consistiam de exame clínico e questionários em que eram avaliadas a dor pela escala analógica visual (EVA) da dor, a satisfação com o procedimento e as complicações. Os exames radiológicos foram realizados no sétimo dia de pós-operatório e após cada retorno.

\section{RESULTADOS}

O seguimento variou de 12,6 a 61,7 meses, sendo em média de 37,5 meses. A comparação entre o grau de cifose média inicial e final entre os sexos encontrase na tabela 2.

TABELA 2

Comparação entre os sexos da evolução do grau de cifose

\begin{tabular}{lccc}
\hline \multicolumn{1}{c}{ Sexo } & $\begin{array}{c}\text { Média cifose } \\
\text { inicial (grau) }\end{array}$ & $\begin{array}{c}\text { Média cifose } \\
\text { final (grau) }\end{array}$ & $\begin{array}{c}\text { \% correção } \\
\text { média }\end{array}$ \\
\hline Masculino & 75,4 & 37,5 & 50,3 \\
Feminino & 83,8 & 31,2 & 62,8 \\
\hline
\end{tabular}

$\mathrm{Na}$ avaliação pré-operatória dos pacientes o grau de cifose torácica variou de $66^{\circ}$ a $94^{\circ}$, com média de $77,6^{\circ}$ (figuras 1 e 2). Após o seguimento pós-operatório, o
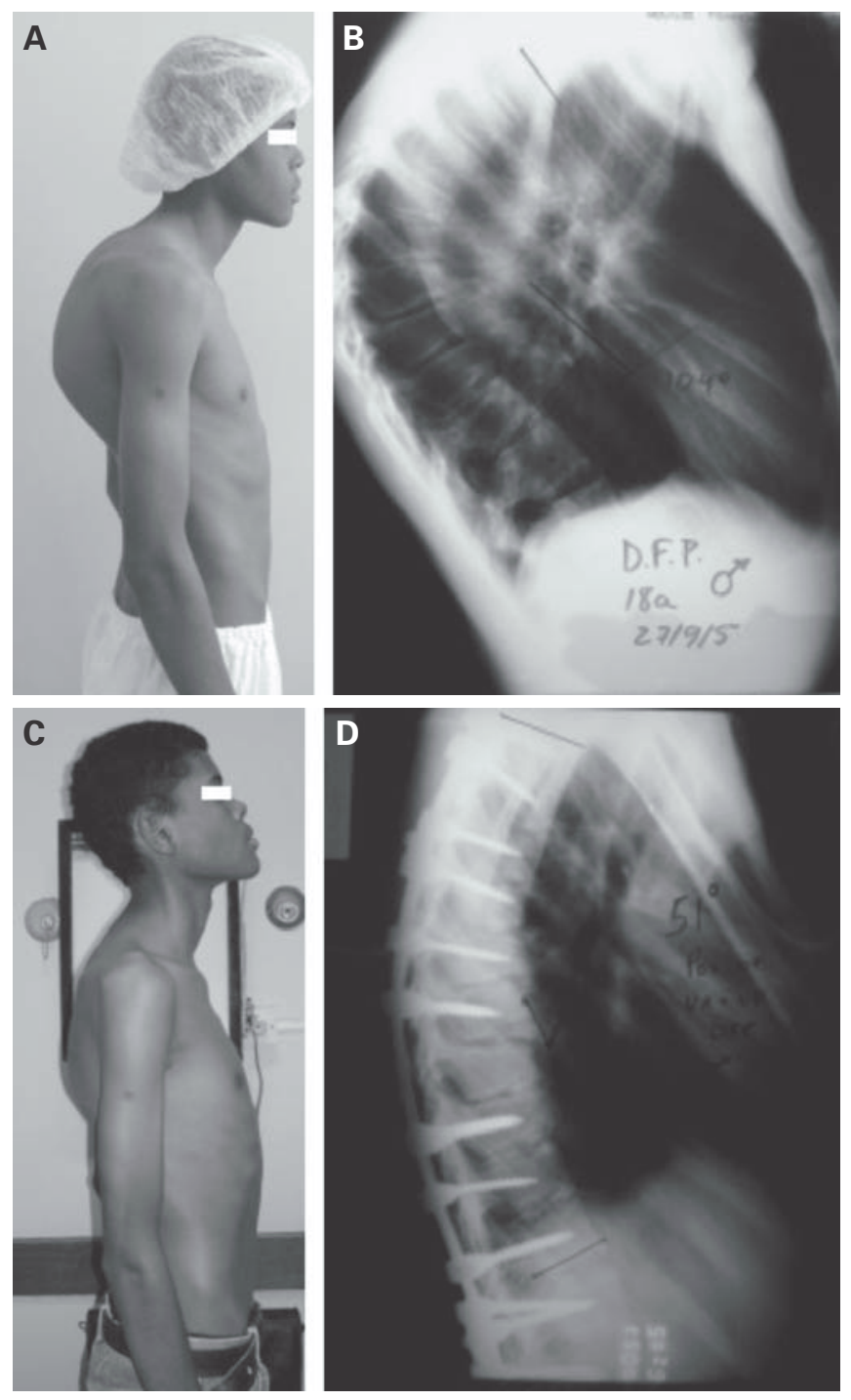

Figura 1 - A) Paciente masculino, 14 anos de idade, imagem préoperatória. B) Radiografia de perfil demonstrando cifose de $104^{\circ}$. C) Imagem do paciente no pós-operatório, demonstrando a meIhora clínica. D) Radiografia de perfil demonstrando a correção para $51^{\circ}$.

grau de cifose aferido nos pacientes foi de $23^{\circ}$ a $50^{\circ}$, com média de $35,8^{\circ}$ (tabela 3 ).

Dos pacientes, 17 apresentavam dor no pré-operatório. O nível da dor foi avaliado pelo método EVA (escala visual analógica), com graus de zero (ausência de dor) a 10 (intensidade máxima de dor). A média obtida na avaliação pré-operatória foi de 6,6 pontos. Após seguimento, os pacientes foram avaliados pelo mesmo método (EVA). Apenas três queixaram-se de 


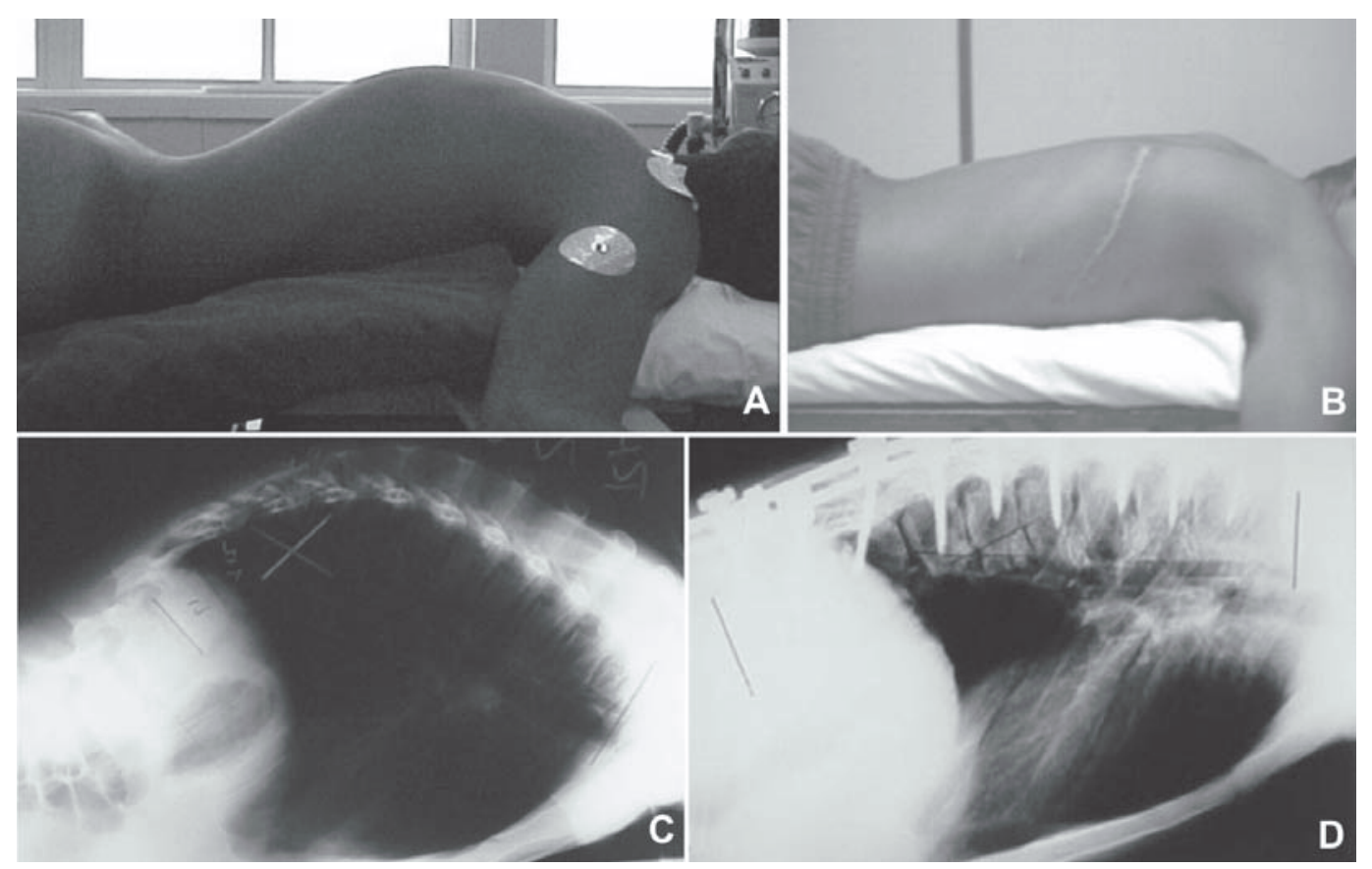

Figura 2 - A) Paciente masculino, 20 anos de idade, imagem pré-operatória. B) Imagem do paciente no pós-operatório, demonstrando a melhora clínica. C) Radiografia de perfil demonstrando cifose de $72^{\circ}$. D) Radiografia de perfil demonstrando a correção para $30^{\circ}$.

TABELA 3

Resultados do tratamento

\begin{tabular}{|c|c|c|c|c|c|c|c|}
\hline Paciente & $\begin{array}{l}\text { Idade } \\
\text { (anos) }\end{array}$ & Sexo & $\begin{array}{l}\text { Cifose } \\
\text { inicial } \\
\text { (grau) }\end{array}$ & $\begin{array}{c}\text { Cifose } \\
\text { final } \\
\text { (grau) }\end{array}$ & $\begin{array}{c}\text { Nível } \\
\text { fusão } \\
\text { posterior }\end{array}$ & $\begin{array}{l}\text { Seguimento } \\
\text { (meses) }\end{array}$ & $\begin{array}{c}\text { № } \\
\text { parafusos }\end{array}$ \\
\hline 1 & 15 & $M$ & 86 & 40 & T2-L2 & 61,7 & 12 \\
\hline 2 & 21 & $M$ & 82 & 48 & T3-L3 & 55,1 & 16 \\
\hline 3 & 33 & $M$ & 80 & 50 & T2-L2 & 48,8 & 20 \\
\hline 4 & 22 & $\mathrm{~F}$ & 72 & 23 & T2-L3 & 48,4 & 20 \\
\hline 5 & 16 & $M$ & 72 & 28 & T4-L2 & 51,9 & 13 \\
\hline 6 & 16 & M & 66 & 30 & T2-L1 & 51,6 & 11 \\
\hline 7 & 18 & M & 90 & 30 & T3-L3 & 46,5 & 18 \\
\hline 8 & 13 & $M$ & 60 & 40 & T4-L2 & 43,9 & 14 \\
\hline 9 & 16 & $\mathrm{~F}$ & 90 & 30 & T3-L2 & 47,6 & 15 \\
\hline 10 & 20 & $M$ & 82 & 40 & T3-L3 & 45,1 & 14 \\
\hline 11 & 17 & $\mathrm{~F}$ & 78 & 32 & T4-L2 & 26,7 & 15 \\
\hline 12 & 15 & $M$ & 65 & 30 & T3-L2 & 36,6 & 16 \\
\hline 13 & 21 & $M$ & 60 & 45 & T2-L2 & 36,2 & 16 \\
\hline 14 & 35 & $M$ & 86 & 48 & T3-L2 & 21,5 & 15 \\
\hline 15 & 16 & $M$ & 69 & 28 & T3-L2 & 18,5 & 19 \\
\hline 16 & 16 & $\mathrm{~F}$ & 94 & 35 & T2-L2 & 25,4 & 16 \\
\hline 17 & 16 & $\mathrm{~F}$ & 85 & 36 & T2-L2 & 14,2 & 16 \\
\hline 18 & 16 & $M$ & 72 & 28 & T2-L2 & 12,6 & 19 \\
\hline 19 & 19 & $M$ & 85 & 40 & T2-L2 & 19,7 & 16 \\
\hline Média & 19,0 & NA & 77,6 & 35,8 & NA & 37,5 & 15,8 \\
\hline
\end{tabular}

Nota: NA: não se aplica; $M=$ masculino; $F=$ feminino. dor residual, sendo a média de pontos no pós-operatório igual a 0,6 .

A satisfação com o procedimento cirúrgico foi de 94,7\% dos pacientes, sendo apenas um paciente insatisfeito. Em relação a um novo procedimento, somente um paciente relatou que não se submeteria novamente à intervenção cirúrgica se houvesse indicação.

No total foram inseridos 301 parafusos (média de 15,8 parafusos por paciente).

Dentre as complicações com os procedimentos foram identificados: um caso de infecção superficial precoce de ferida operatória tratada clinicamente; um caso de quebra de dois parafusos assintomático, sendo um achado radiológico; um caso de infecção tardia com 24 meses de pós-operatório que se resolveu com a retirada do implante, sem perda da correção da deformidade; um caso de soltura do implante distal que necessitou nova intervenção cirúrgica com ampliação do nível da artrodese; e três pacientes com queixa de dor residual, sendo dois com relato de dores esporádicas que não necessitaram qualquer tratamento. 


\section{DISCUSSÃO}

$\mathrm{Na}$ CS eventualmente é necessário o tratamento cirúrgico, mas alguns pontos ainda permanecem em debate no momento da indicação dos procedimentos. Desde que Bradford et al fizeram o primeiro relato sobre fusão posterior no tratamento da CS, as indicações cirúrgicas bem como as técnicas operatórias vêm apresentando grandes modificações e, em varias séries de casos publicados, dor e deformidade são tidas como os critérios adotados para se optar pela indicação cirúrgica ${ }^{(10-12)}$. Alguns autores indicam cirurgia para os casos de dor refratária ao tratamento conservador ${ }^{(13-}$ 14). Lowe recomenda tratamento cirúrgico na doença de Scheuermann em adultos com cifose maior do que ou igual a $75^{\circ}$, com quadro de dor persistente apesar do tratamento conservador, e naqueles com deformidades que influenciam a aparência estética do paciente ${ }^{(13)}$. Murray et al reservam a opção cirúrgica para os casos com dor e deformidade inaceitáveis ${ }^{(15)}$.

Considerando os critérios utilizados no presente estudo, fomos condizentes com a literatura quando avaliamos o grau de deformidade associado às queixas de dor do paciente após tentativas de tratamento conservador para a indicação do tratamento cirúrgico na CS. $\mathrm{Na}$ nossa série de pacientes reservamos a indicação do tratamento cirúrgico para os com queixa de dor refratária ao tratamento conservador ou deformidade inaceitável do ponto de vista estético.

Nos 22 pacientes revisados por Bradford et al, submetidos à fusão espinhal por via anterior/posterior e instrumentação de Harrington, a média de correção da cifose foi de $32,8 \%$, com seguimento de 35 meses $^{(16)}$. Otsuka et al, numa série de 10 pacientes tratados com a mesma técnica de Bradford, obtiveram correção média da cifose de 44,9\% ${ }^{(14)}$. Herndon et al, em 13 pacientes, obtiveram correção média de $41 \%$, após seguimento médio de 29 meses $^{(11)}$. Taylor et al efetuaram fusão vertebral posterior com material de Harrington em 27 pacientes com correção média de $27 \%$, com seguimento de 28 meses. Dentre as complicações maiores foram citadas duas falências do implante que necessitaram nova intervenção. Das complicações associadas a risco grave ao paciente, foram relatados um pneumotórax hipertensivo e um caso de obstrução do trato gastrointestinal alto que requereu hiperalimentação por 10 semanas $^{(12)}$. Bradford et al examinaram 24 pacientes com fusão espinhal anterior/posterior com Harrington. A correção média foi de $36,4 \%$, com seguimento médio de 39 meses. Complicações maiores incluíram uma infecção profunda, uma pseudartrose que necessitou enxerto ósseo, uma cifose juncional que necessitou extensão da artrodese, uma úlcera de pressão com tratamento pela cirurgia plástica, dois implantes dolorosos requerendo sua remoção e três deiscências de ferida operatória parcial que tiveram de ser submetidas a reparo. Complicações com risco de morte incluíram duas embolias pulmonares, uma obstrução do trato gastrointestinal alto que foi submetida à laparotomia e o mesmo paciente com hemotórax persistente, que requereu decorticação pleural ${ }^{(10)}$.

Embora esses e outros estudos mostrem que as cirurgias com instrumentação de Harrington são eficazes, freqüentemente se observam complicações de gravidade maior. Além disso, apresentam um grau inferior quando comparado com os implantes de segunda e terceira geração.

Utilizando a instrumentação de Cotrel-Dubousset em oito pacientes com CS, de Jonge et al descreveram correção de $49 \%$, apresentando no pós-operatório dois pacientes com dor crônica refratária ao tratamento cirúrgico ${ }^{(17)}$.

Lee et al compararam 18 pacientes com doença de Scheuermann submetidos à fusão espinhal posterior com uso de parafuso pedicular com 21 pacientes em que foi realizada fusão anterior/posterior combinada com emprego de construções utilizando ganchos ou parafusos/ganchos. No primeiro grupo, o grau médio de correção foi de $52 \%$, após seguimento médio de 31,7 meses, enquanto no segundo grupo de pacientes a média foi de $54,6 \%$, após seguimento médio de 67,5 meses. Dos 18 pacientes do primeiro grupo, nove tiveram complicações, enquanto oito dos 21 pacientes do segundo grupo apresentaram complicações como cifose funcional proximal e distal, paraplegia imediatamente após o procedimento posterior, tendo-se procedido à retirada emergencial do material ${ }^{(18)}$. 
A evolução da técnica e dos materiais de implante, em especial o sistema de parafusos pediculares, permitiu, além de maior correção da cifose, diminuição do número de complicações. Os resultados obtidos no presente estudo descrevem correção média de 53,8\%, alto índice de satisfação com o procedimento e melhora do quadro álgico. O número de pacientes avaliados e os resultados são similares aos observados na literatura. Observou-se baixo índice de complicações menores: um caso de infecção superficial e precoce de ferida operatória tratada clinicamente; um caso de infecção tardia com 24 meses de pós-operatório que se resolveu com a retirada dos implantes, sem perda da correção da deformidade; um caso de soltura dos implantes distais no segundo mês de pós-operatório que necessitou de nova intervenção com ampliação da instrumentação; e três pacientes com queixa de dor residual, sendo dois com relato de dores esporádicas que não necessitaram de qualquer tratamento. Nenhuma complicação de maior gravidade foi observada.

Descrevemos também a nossa técnica de passagem de parafuso a mão livre ( free hand) através do uso de broca e trepano de baixa rotação, a qual difere da técnica descrita por Kim et $a l^{(9)}$.

Em 27 pacientes com hipercifose do adolescente, Johnston et al utilizaram a abordagem posterior isola-

\section{REFERÊNCIAS}

1. Scheuermann HW. Kyphosis dorsalis juvenilis. Orthop Chir. 1921;41:305.

2. Sorensen KH. Scheuermann's juvenile kyphosis. Copenhagen, Munksgaard; 1964.

3. Bradford DS. Juvenile kyphosis. In: Lonstein JE, Bradford DS, Winter RB, Ogilvie J. Moe's Textbook of scoliosis and other spinal deformities. $3^{\text {rd }}$ ed; 1995. p. 349-67.

4. Lim M, Green DW, Billinghurst JE, Huang RC, Rawlins BA, Widmann RF, et al. Scheuermann kyphosis: safe and effective surgical treatment using multisegmental instrumentation. Spine. 2004;29(16):1789-94. Erratum in: Spine. 2004;29(19): 2198.

5. Ascani E, La Rosa G, Ascani C. Scheuermann kyphosis. The pediatric spine. $2^{\text {nd }}$ ed. Stuart L. Weinstein; 2001. p. 431.

6. Sachs B, Bradford D, Winter R, Lonstein J, Moe J, Willson S. Scheuermann kyphosis. Follow-up of Milwaukee-brace treatment. J Bone Joint Surg Am. 1987;69(1):50-7. da para artrodese com o instrumental de haste, parafuso e ganchos em 20 pacientes e a dupla abordagem anterior e posterior em sete pacientes. Não houve diferença estatisticamente significativa entre os grupos em relação ao total de correção (53\% contra $46 \%$, respectivamente). Dentre as complicações, foi relatada uma fratura de haste assintomática ${ }^{(19)}$.

Quanto à utilização da via posterior isolada com sistema de parafusos pediculares e osteotomias no tratamento da cifose de Scheuermann, bons resultados vêm sendo publicados na literatura, mas destacamos que a casuística ainda é pequena e existe a necessidade de maior seguimento dos casos no pós-operatório, bem como novos estudos para avaliar o real papel da instrumentação posterior unicamente como método de tratamento da cifose de Scheuermann.

\section{CONCLUSÃO}

Nesta série de pacientes submetidos à discectomia e artrodese anterior por toracotomia seguida por artrodese por via posterior e instrumentação com sistema de parafusos pediculares, os resultados foram satisfatórios quanto ao percentual de correção, à satisfação do paciente e à melhora do quadro de dor, com baixo risco de complicações.

7. Suk SI, Kim WJ, Lee SM, Kim JH, Chung ER. Thoracic pedicle screw fixation in spinal deformities: are they really safe? Spine. 2001;26(18):2049-57.

8. Macedo RD, Fontes BPC, Cunha FM, Werlang PM. Sistema de parafusos pediculares no tratamento de deformidades vertebrais: analise da correção e das complicações imediatas. Rev Bras Ortop. 2006;41(10):417-24.

9. Kim YJ, Lenke LG, Bridwell KH, Cho YS, Riew KD. Free hand pedicle screw placement in the thoracic spine: is it safe? Spine. 2004;29(3):333-42.

10. Bradford DS, Ahmed KB, Moe JH, Winter RB, Lonstein JE. The surgical management of patients with Scheuermann's disease: a review of twenty-four cases managed by combined anterior and posterior spine fusion. J Bone Joint Surg Am. 1980;62(5):705-12.

11. Herndon WA, Emans JB, Micheli LJ, Hall JE. Combined anterior and posterior fusion for Scheuermann's kyphosis, Spine. 1981;6(2):125-30. 
12. Taylor TC, Wenger DR, Stephen J, Gillespie R, Bobechko WP. Surgical management of thoracic kyphosis in adolescents. J Bone Joint Surg Am. 1979;61(4):496-503.

13. Lowe TG. Double L-rod instrumentation in the treatment of severe kyphosis secondary to Scheuermann's disease. Spine. 1987;12(4):336-41.

14. Otsuka NY, Hall JE, Mah JY. Posterior fusion for Scheuermann's kyphosis. Clin Orthop Relat Res. 1990;(251): $134-9$.

15. Murray PM, Weinstein SL, Spratt KF. The natural history and long-term follow-up of Scheuermann kyphosis. J Bone Joint Surg Am. 1993;75(2):236-48.
16. Bradford DS, Moe JH, Montalvo FJ, Winter RB. Scheuermann's kyphosis. Results of surgical treatment by posterior spine arthrodesis in twenty-two patients. J Bone Joint Surg Am. 1975;57(4):439-48.

17. de Jonge T, Illés T, Bellyei A. Surgical correction of Scheuermann's kyphosis. Int Orthop. 2001;25(2):70-3.

18. Lee SS, Lenke LG, Kuklo TR, Valenté L, Bridwell KH, Sides B, et al. Comparison of Scheuermann kyphosis correction by posterior-only thoracic pedicle screw fixation versus combined anterior/posterior fusion. Spine. 2006;31(20):2316-21.

19. Johnston $\mathrm{CE} 2^{\text {nd }}$, Elerson E, Dagher G. Correction of adolescent hyperkyphosis with posterior-only threaded rod compression instrumentation: is anterior spinal fusion still necessary? Spine. 2005;30(13):1528-34.

Declaração de inexistência de conflitos de interesse: Declaramos para os devidos fins que não há qualquer conflito de interesse que impeça a publicação deste artigo. 